\title{
Live cell vaccines expressing B7.1, monocyte chemoattractant protein 1 and granulocyte-macrophage colony stimulation factor derived from mouse HPV16-transformed cells
}

\author{
MONIKA LAKATOSOVÁ-ANDELOVÁ ${ }^{1}$, PAVEL JINOCH ${ }^{1}$, MARTINA DUSKOVÁ ${ }^{1}$, \\ IURI MARINOV ${ }^{2}$ and VLADIMÍR VONKA ${ }^{1}$ \\ ${ }^{1}$ Department of Experimental Virology, ${ }^{2}$ Laboratory of Flow Cytometry, Institute of \\ Haematology and Blood Transfusion, U Nemocnice 1, Prague, The Czech Republic
}

Received July 27, 2007; Accepted September 21, 2007

\begin{abstract}
One of the gene therapy strategies in oncology is immunization with cancer cells that express various cytokines. We used a thymidine-kinase deficient (cTK') cell line designated 123IA, which had been derived from HPV16transformed mouse (C57BL/6) cells MK16/I/III/ABC (MK16). To obtain genetically modified cells, 123IA cells were transfected with bicistronic plasmid vectors carrying the herpes simplex type 1 thymidine kinase (HSV TK) gene and either the gene for the mouse B7.1 (CD80) co-stimulatory molecule or the gene for the monocyte-chemoattractant protein 1 (MCP-1). For control purposes, a plasmid vector carrying only the HSV TK gene was used. The transfected cells were cultivated in medium supplemented with hypoxanthine, aminopterin and thymidine. For comparative purposes we also used B9 cells, which express the granulocyte-macrophage colony stimulation factor (GM-CSF) and had been derived from 123A cells by transduction with the recombinant adeno-associated virus carrying the HSV TK gene and the mouse GM-CSF gene. All of the cell lines isolated were found to be sensitive to minute amounts of ganciclovir, revealing the production of HSV TK, and to express the respective transgenes. When inoculated into 5-week-old female syngeneic mice, cells expressing either GM-CSF or B7.1 were non-oncogenic. On the other hand, nearly all mice inoculated with MCP-1-producing cells developed tumours, though considerably later than animals inoculated with the same dose of the parental MK16 cells. Animals injected with GM-CSF- or B7.1-producing cells were protected against challenge with the parental MK16 cells.
\end{abstract}

Correspondence to: Dr Vladimír Vonka, Department of Experimental Virology, Institute of Haematology and Blood Transfusion, U Nemocnice 1, Prague, The Czech Republic E-mail: vladimir.vonka@uhkt.cz

Key words: cancer vaccines, HPV16, herpes simplex type 1 thymidine kinase, B7.1, monocyte-chemoattractant protein 1, granulocyte-macrophage colony stimulation factor, gene-modified tumour cells
When another mouse (C57BL/6) HPV16-transformed oncogenic cell line, TC-1, which differs from the MK16 cells in a number of properties such as MHC class I and B7.1 expression, was used for the challenge, the protective effect was much less pronounced.

\section{Introduction}

Genetic immuno-modification of tumour cells may be used as a cancer immunotherapy strategy $(1,2)$. Modified tumour cells can directly and efficiently stimulate anti-tumour immune responses. Genes for different cytokines and chemokines have been used in this kind of experiments with either in situ or, much more frequently, in vitro cultivated tumour cells as their recipients. Our laboratory and a collaborating one have used HPV16 transformed mouse cells for this purpose and have shown that their permanent genetic modification (3-5) or even a transient expression of the immunostimulatory products after infection with gene-modified viruses expressing various cytokines (6) can abolish their oncogenicity and increase their immunogenicity. These experiments were aimed at providing more information on immune responses to cells that express the HPV E6 and E7 oncoproteins, which might possibly be utilized in the development of therapeutic vaccines against $\mathrm{HPV}$-induced cancers in the future. In the present series of experiments we continued in these efforts and below report on the properties of gene-modified HPV16 cells that express either the B7.1 co-stimulatory molecule or MCP-1. These cells were compared with cells that had been previously derived from the same parental cells but express GM-CSF (7).

\section{Materials and methods}

Cell lines and media. The MK16 cell line was derived in our laboratory by transformation of mouse (C57BL/6) kidney cells via co-transfection with the HPV16 E6 and E7 genes and the activated H-ras oncogene (8). TC-1 cells (obtained from T.C. $\mathrm{Wu}$, Johns Hopkins University, Baltimore, MD, USA) were isolated after transfection of mouse C57BL/6 lung cells with a similar set of genes (9). The two cell lines differ in a number of characteristics. The TC-1 cells have fibroblastoid morphology, do not express cytokeratines and express MHC class I 
and B7.1 co-stimulatory molecules on their surfaces (10). They are highly oncogenic $\left(1 \mathrm{TID}_{50}\right.$ corresponds to approximately $1 \times 10^{4}$ cells), but subcutaneous tumours induced by them do not metastasize spontaneously. On the other hand, MK16 cells have epitheloid morphology (8), do form cytokeratins and are MHC class I and B7.1 negative. They are less oncogenic ( $1 \mathrm{TID}_{50}$ corresponds to $1 \times 10^{5}$ cells) than the TC- 1 cells but the subcutaneous tumours induced by them do metastasize spontaneously to lymph nodes and lungs. It has also been shown that the MK16 cells produce MHC class I molecules when cultivated in the presence of interferon $\gamma$ as well as in vivo in the course of tumour growth (11). Owing to the expression of MHC class I molecules, the MK16 cells have acquired sensitivity to effector cytolytic lymphocytes from immunized animals. A thymidine kinase negative (cTK') subline of MK16 cells, designated 123, has been derived after repeated passages in the presence of increasing concentrations of 5-bromo-2deoxyuridine (Sigma-Aldrich Corp., St. Louis, MO, USA). The 123IA subline used in the present experiments has been isolated from a tumour induced by 123 cells after cultivation of these cells in a medium supplemented with $100 \mu \mathrm{g} \mathrm{BrdU} / \mathrm{ml}$ and has since been cultivated in the same medium. B9 cells have been derived by transduction of 123IA cells with a recombinant adeno-associated virus that carries the HSV TK gene and the mouse GM-CSF gene and express relatively high levels of this cytokine (>100 ng/106 cells/48 h) (7). The CaSki cell line is positive for HPV16 DNA (12) and was used as an HPV DNA-positive control when the products of polymerase chain reaction (PCR) tests were analysed (13). RMA is a mutagenized cell subline derived from Rauscher leukaemia virus-induced lymphoma RBL-5 of C57BL/6 origin (14). The RMA-derived transfectant clone $2 \mathrm{~F} 11$, expressing the $\mathrm{E} 7$ of HPV16 (15) was used in cytotoxicity tests.

All cell lines, except RMA and 12F1, were cultivated in Dulbecco's modified Eagle's medium (DMEM, BRL, Paisley, UK) with 10\% FCS (PAA Laboratories, Linz, Austria), 100 U/ $\mathrm{ml}$ penicillin, $100 \mu \mathrm{g} / \mathrm{ml}$ streptomycin and $2 \mathrm{mM}$ L-glutamine (Sevac, Prague, The Czech Republic), at $37^{\circ} \mathrm{C}$ in a $5 \% \mathrm{CO}_{2}$ atmosphere. In the case of transduced cells, the medium was supplemented with hypoxanthine, aminopterin and thymidine (HAT), while 123IA cells were cultivated in the presence of $100 \mu \mathrm{g} / \mathrm{ml}$ of BrdU. RMA and $12 \mathrm{~F} 1$ cells were cultivated in RPMI-1640 medium (Sigma-Aldrich Corp.) supplemented with $10 \%$ FCS, 2 mM L-glutamine and antibiotics.

Plasmids and transfection experiments. The pUF2-MCP-1/ IRES/HSVTK, pUF2-B7.1/IRES/HSVTK and pTR-IRES/ HSVTK plasmids constructed previously in our laboratory (10) were propagated in the E. coli SURE strain in LB broth medium containing $100 \mu \mathrm{g} / \mathrm{ml}$ ampicillin and were purified using the Qiagen Plasmid Maxi kit (Qiagen, Germany). Counts of $3 \times 10^{5}$ 123IA cells were seeded into plates. The next day the cultures were transfected with $12 \mu \mathrm{g}$ DNA in HEPES buffered saline solution using modified calcium phosphate precipitation (16). After 24-h incubation the regular medium was replaced by DMEM supplemented with hypoxanthine, aminopterin and thymidine (HAT Media Supplement, Invitrogen, Carlsbad, CA, USA). After 14-day cultivation the cells were harvested. Subsequently, cell clones were isolated and expanded in HAT medium.
ELISA. Counts of $5 \times 10^{5}$ cells in $4 \mathrm{ml}$ medium were seeded in 6- $\mathrm{cm}$ dishes. The MCP-1 concentration in the medium was measured after 48-h incubation using a commercially available MCP-1 OPTEIA ${ }^{\mathrm{TM}}$ kit (Pharmingen, San Diego,CA,).

Flow cytometry. Counts of $0.5 \times 10^{6}$ trypsinized cells were washed twice with PBS. MHC class I molecules were detected after incubation of the cells with anti-mouse $\mathrm{H}-2 \mathrm{~K}^{\mathrm{b}} / \mathrm{H}-2 \mathrm{D}^{\mathrm{b}}$ monoclonal antibody (Pharmingen) or with isotype control antibody (Sigma, St. Louis, MO) at $4^{\circ} \mathrm{C}$ for 20-30 min. The cells were then washed in PBS and incubated with FITCconjugated goat anti-mouse IgG antibody (Pharmingen). B7.1 molecules were detected after incubation of cells with FITClabelled anti-mouse CD80 monoclonal antibody or isotype control antibody (Pharmingen). Fluorescence was measured with a flow cytometer (FACSCalibur, Beckman Dickinson). MK16 cells (which are both MHC class I and B7.1 negative) and TC1 cells (which are both MHC class I and B7.1 positive) were used as negative and positive controls, respectively.

Ganciclovir sensitivity assay. Cells were seeded in a number of $5 \times 10^{2}$ cells per well in $100 \mu 1$ culture medium in 96-well plates (TPP, Trasadingen, Switzerland). Sensitivity to ganciclovir (GCV) was tested using the CellTiter 96R Aqueous non-radioactive cell proliferation assay (Promega, Madison, WI). The GCV (Cymevene; Roche, Basel, Switzerland) concentrations used ranged from 0.8 to $200 \mu \mathrm{M}$. HSV TKpositive B9 cells (7) and HSV TK-negative 123IA cells were used as controls. The survival rate was measured after 5-day incubation. The $50 \%$ inhibition concentration $\left(\mathrm{IC}_{50}\right)$ was calculated by non-linear regression analysis of the sigmoid curve fitted with variable slopes to normalized data using Graphpad PRISM 4.0 software (Graphpad Software Inc. San Diego, CA).

RT-PCR. Total RNA was extracted from the cells cultured at $70-80 \%$ confluence in $10-\mathrm{cm}$ culture dishes. The RNAeasy mini kit (Qiagen GmbH, Hilden, Germany) was used for this purpose. The reverse transcriptase-polymerase chain reaction (RT-PCR) and the subsequent analysis of its products were performed as described previously (13). A positive control reaction was performed with $100 \mathrm{ng}$ DNA isolated from CaSki cells.

Animals and oncogenicity and immunogenicity tests. Six- to eight-week-old female C57BL/6 mice $\left(\mathrm{H}-2^{\mathrm{b}}\right)$ were obtained from Charles River, Germany. All experiments were carried out in accordance with the Guidelines for Animal Experimentation valid in the Czech Republic. For oncogenicity tests, cells were trypsinized and washed three times with PBS. Counts of $5 \times 10^{6}$ MK16 cells and the gene-modified cells in $150 \mu 1$ volumes of PBS were injected subcutaneously (s.c.) into the right flanks of mice. Starting at one week later, mice were monitored for the appearance of tumours. Tumour diameters were measured with a calliper twice a week. For the immunization experiments the animals received a second s.c. dose of the same size two weeks after the first one, injected into the opposite flank. Two weeks after the second dose the mice were challenged with $5 \times 10^{5}$ parental MK16 cells or with 
$5 \times 10^{4} \mathrm{TC}-1$ cells administered s.c. into the interscapular area. Tumour growth was monitored twice a week. Animals were euthanized when their tumour size reached $15 \mathrm{~mm}$.

Chromium release cytotoxicity assay. At the time of challenge splenocytes from three animals of each immunized and the naive group were pooled and $2 \times 10^{6}$ cells were cultivated for 5 days in $1 \mathrm{ml}$ of complete RPMI-1640 medium supplemented with $6 \mu \mathrm{g}$ of the RAHYNIVTF peptide (carrying the HPV16 E7-specific H-2D b epitope, aa 49-57). The cytotoxicity of splenocytes was measured in terms of ${ }^{51} \mathrm{Cr}$ release from target cells (2F11 or RMA cells). Target cells (counts of $2 \times 10^{6}$ ) were labelled with $100 \mu \mathrm{Ci}{ }^{51} \mathrm{Cr}$ for $90 \mathrm{~min}$ and washed 3 times with complete RPMI medium. Then $1 \times 10^{3}$ labelled cells were distributed per well (in triplicate) and incubated with the effector cells using effector/target cell ratios of 80, 40, 20 and 10. The percentage of specific lysis was calculated as follows:

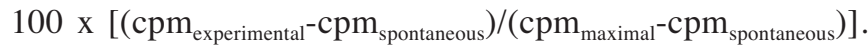
Maximum ${ }^{51} \mathrm{Cr}$ release was determined by lysis of target cells in $2 \%$ Triton X-100. Spontaneous release was determined in target cells incubated in the absence of effector cells.

Statistical methods. Statistical evaluation of tumour growth was made by two-way analysis of variance (ANOVA). A difference between groups was considered statistically significant when $\mathrm{p}<0.05$ in the Graphpad PRISM 4.0 programme (Graphpad software Inc.)

\section{Results}

Generation of genetically modified cell lines. 123IA cells were transfected separately with the pUF2-MCP-1/IRES/

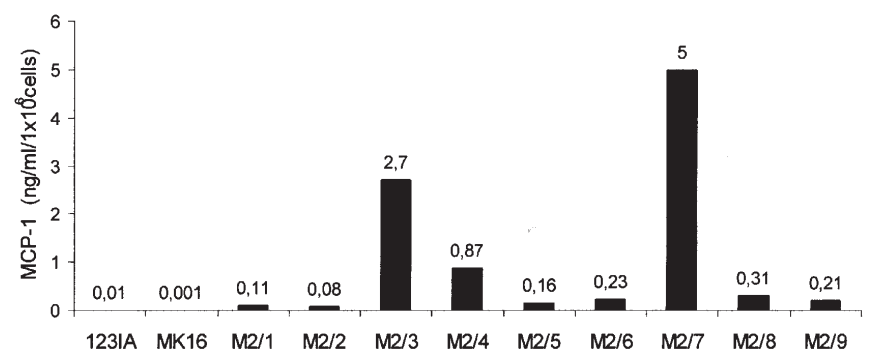

Figure 1. Production of MCP-1 by individual cell clones. The 123IA and MK16 cells were used as negative controls. Samples were removed after $48 \mathrm{~h}$ of cultivation in DMEM/HAT medium.

HSVTK, pUF2-B7.1/IRES/HSVTK or pTR-IRES/HSVTK plasmid, or mock-transfected, and maintained in medium containing HAT. Within two weeks several colonies appeared in plates in which the transfected cells had been seeded, whereas no mock-transfected cells survived. Cells from the HAT-resistant colonies were isolated and cloned in 96-well plates in HAT-supplemented medium. From each cell line several clones were isolated, tested for the respective transduced gene product, and the most efficient producers were selected for further tests. The results obtained with the clones isolated from the pUF2-MCP-1/IRES/HSVTK-transfected cell population and with the control cells are shown in Fig. 1. It is evident that the individual clones differed markedly in MCP-1 production. For further analysis, the M2/7 clone was chosen. Clones selected after transfection with pUF2B7.1/IRES/HSVTK were tested by flow cytometry. The clones derived from the transfected cultures expressed the costimulatory molecule more efficiently than the 'naturally'
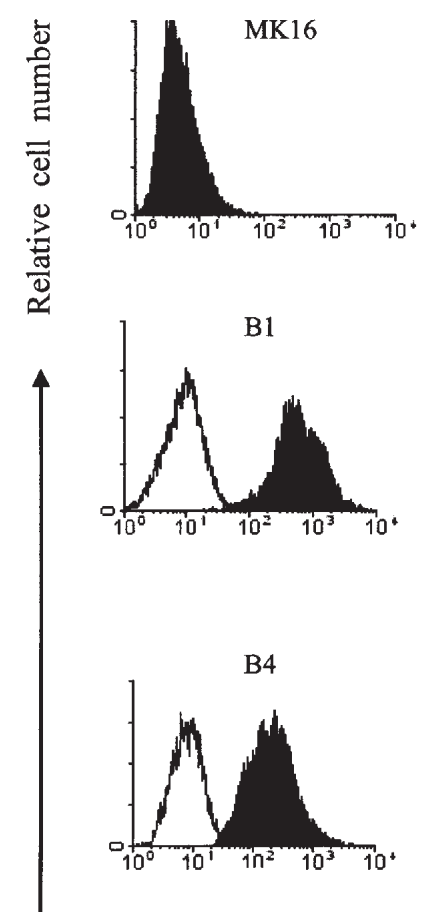
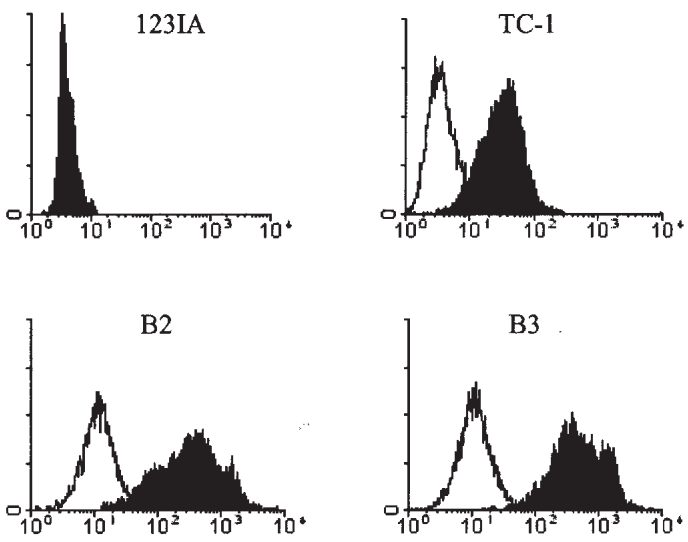

B5

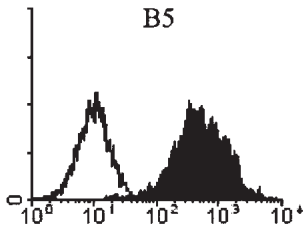

\section{Log fluorescence}

Figure 2. Expression of B7.1 (filled curve) on gene-modified cells (B1 to B5) in comparison with the parental 123IA cells and the MK16 cells as determined by flow-cytometry. TC-1 cells were used as a positive control. Solid line represents isotype control. 


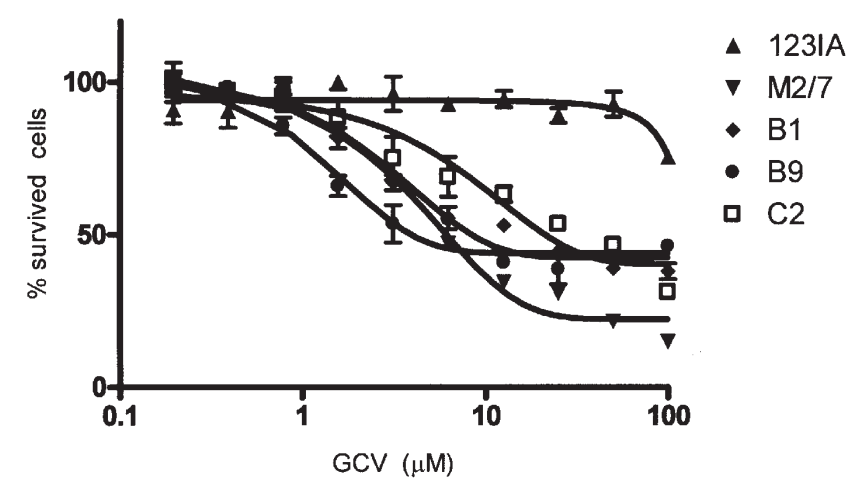

Figure 3. In vitro GCV sensitivity of the M2/7, B1, B9, C2 cells and 123IA cells, estimated according to the CellTiter 96 Aqaeous non-radioactive cell proliferation assay. The data represent means of the three independent experiments (error bars, $\pm \mathrm{SD}$ ).

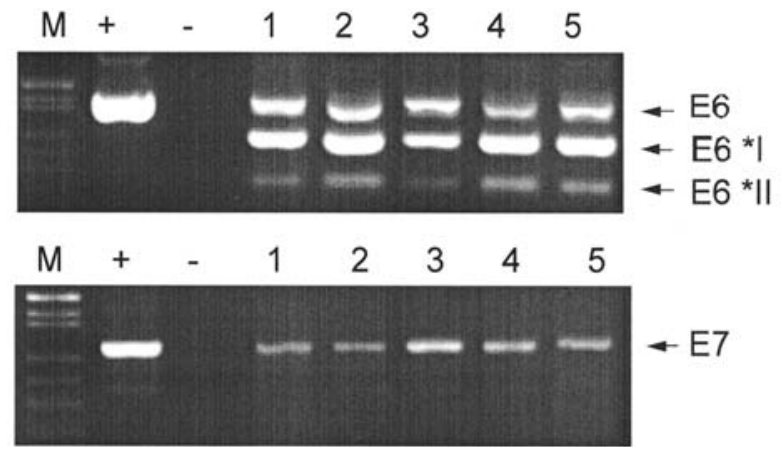

Figure 4. Expression of the HPV16 E6 and E7 genes in (1) MK16, (2) C2, (3) $\mathrm{M} 2 / 7$, (4) B1 and (5) B9 cells as determined by RT-PCR. DNA extracted from HPV16-transformed CaSki cells and the sample containing sterile water served as a positive and a negative control, respectively. M, molecule weight determined with plasmid pUC18 fragments (cleavage with Msp I).

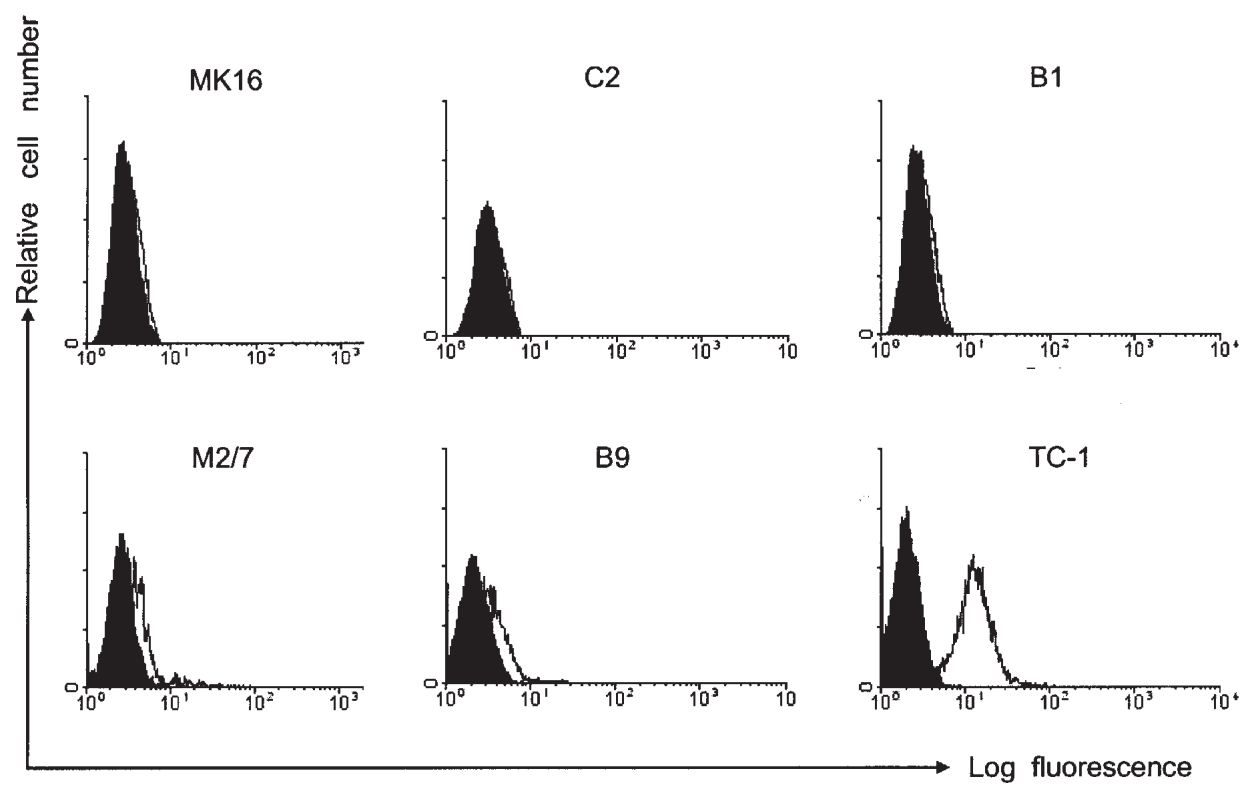

Figure 5. Expression of MHC class I molecules (solid line) on gene-modified cells. MK16 and TC-1 cells were used as a negative and a positive control, respectively. Filled curve represents isotype control.

positive TC-1 cells and the clones designated B1 and B5 appeared to be the most efficient producers (Fig. 2). The B1 clone was singled out for further tests. The production of MCP-1 and B7.1 in the selected clones was stable over eight passages, both in the presence and absence of HAT (results not shown).

Large stocks of the selected cell lines were frozen and kept in liquid nitrogen. Similar stocks were prepared from the GM-CSF-producing B9 cells isolated earlier and from the $\mathrm{C} 2$ clone randomly selected from the culture transduced with pTR-IRES/HSVTK plasmid. In all subsequent experiments the third passages of thawed cells were used.

GCV sensitivity of $M 2 / 7, B 1, B 9$ and $C 2$ cells. To ensure that the cell lines selected did not represent revertants to the $\mathrm{cTK}^{+}$ phenotype, which would easily grow in HAT media, we tested them for sensitivity to GCV. The results obtained with all four of the gene-modified cell lines and the 123IA cells, which served as an HSV TK-negative control, are shown in Fig. 3. Clearly, the B9 cells and the newly transduced cells were highly sensitive to GCV. Their $\mathrm{IC}_{50}$ value $(50 \%$ inhibition of proliferation) was in the range of 1.5 to $5.5 \mu \mathrm{M}$, while the HSV TK-negative 123IA cells were only slightly inhibited at the highest GCV concentration used $(100 \mu \mathrm{M})$. These results indicated that all four of the transduced lines tested expressed HSV TK.

Expression of HPV16 E6/E7 genes in transfected cells. B9, $\mathrm{B} 1, \mathrm{M} 2 / 7$ and C2 cells were tested for the expression of E6 and E7 by RT-PCR (Fig. 4). DNA extracted from CaSki cells served as a positive control. In all of the gene-modified cell lines tested three transcripts of the HPV16 E6 gene (unspliced, $420 \mathrm{bp}$; and two spliced, 238 and $121 \mathrm{bp}$ ) and a single transcript of the HPV16 E7 gene were present. Thus, this assay did not reveal any difference between the original MK16 cells and the transduced cells. 


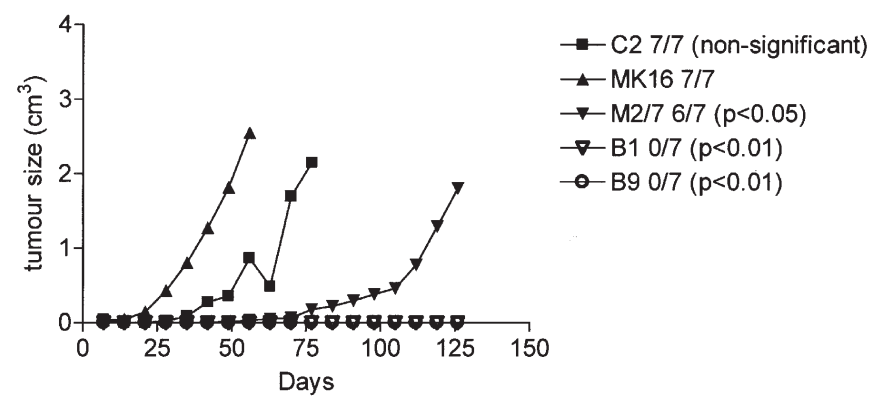

Figure 6. Tumour growth in mice after inoculation of gene-modified and MK16 cells. Cells $\left(5 \times 10^{6}\right)$ were inoculated into the right flank. Numbers $/ 7$ represent the rate of tumour-positive mice.

A)

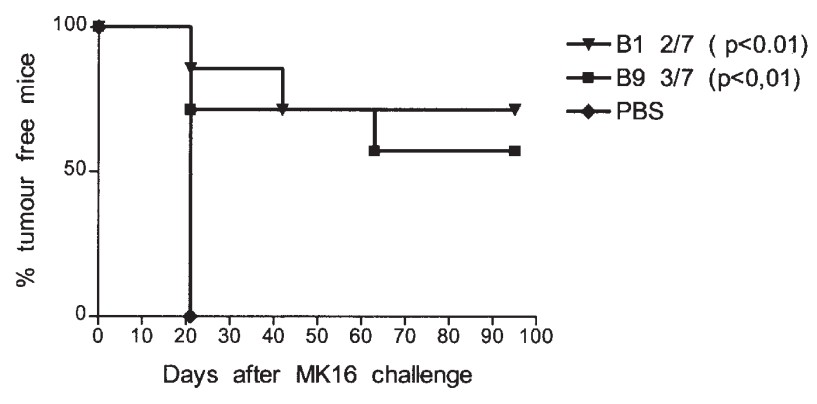

B)

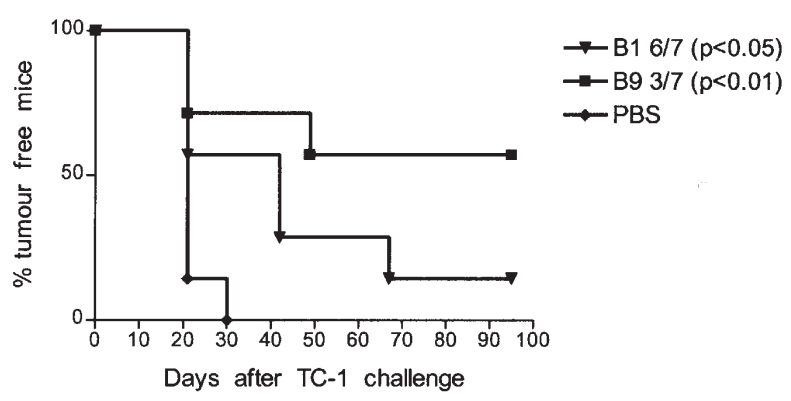

Figure 7. Immunization/challenge experiment. Mice were immunized with two doses $\left(5 \times 10^{6}\right.$ cells) of B9 or B1 cells and challenged with either $5 \times 10^{5}$ MK16 cells (A) or $10^{4}$ TC-1 cells (B). Control mice received PBS. Numbers $/ 7$ represent the rate of tumour-positive mice.

Expression of MHC class 1. As already mentioned, MK16 cells have a downregulated in vitro expression of MHC class I molecules. We tested the expression of MHC class I in transduced cells derived from 123IA, in comparison to the MK16 cells used as a negative control (Fig. 5). The genetic modification was not associated with a marked expression of MHC class I molecules. Some positivity was observed in B9 cells and, to a lesser extent, in M2/7 cells. In both of these cell lines, the quantities of these molecules were much lower than in the MHC class I-positive TC-1 cells.

Oncogenicity tests. Tumour development after injection of $5 \times 10^{6}$ cells of all four of the gene-modified cell lines is shown in Fig. 6. The MK16 cells were used as a positive

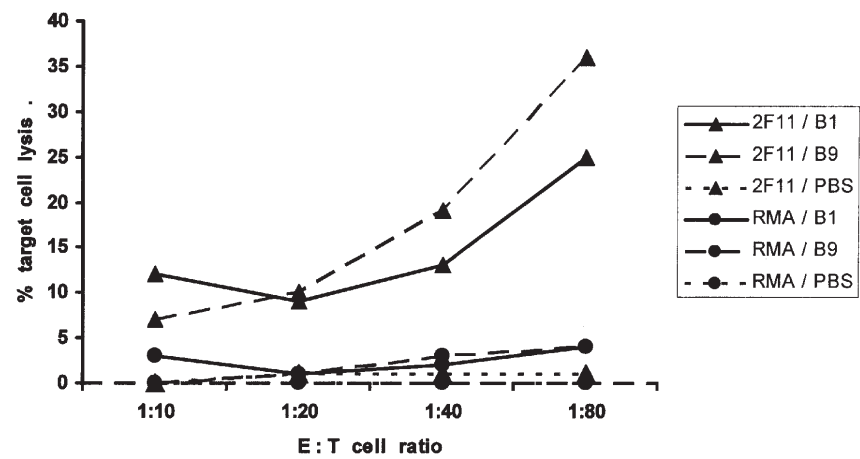

Figure 8. In vitro cytotoxicity of spleen cells isolated from mice immunized with B1 or B9 cells and from control (PBS) animals. Pooled splenocytes from three mice which were sacrificed at the time of challenge.

control. In agreement with the earlier experiments (7), B9 cells showed no oncogenic activity. Neither did B1 cells, which express B7.1 co-stimulatory molecule on their surfaces, induce tumours. On the other hand, all animals inoculated with $\mathrm{C} 2$ cells developed tumours, though at a somewhat slower rate than animals inoculated with the MK16 cells, but the difference was not statistically significant. Nearly all animals (6 out of 7) inoculated with M2/7 cells also developed tumours, but significantly later than animals that had been injected with the MK16 cells. Similar results were obtained in repeated tests (data not shown).

Immunization/challenge experiments. To test the immunogenicity of the gene modified cells, mice which had received two $5 \times 10^{6}$ doses of either B9 or B1 cells at a two week interval, were challenged with either $5 \times 10^{5}$ MK16 cells or $5 \times 10^{4} \mathrm{TC}-1$ cells. The immunizing efficiency of both cell lines was nearly equal, if MK16 cells were used for the challenge (Fig. 7A). In both instances the majority of mice were protected. However, where TC-1 cells were used for the challenge, the protection effect was much lower in mice immunized with B1 cells (Fig. 7B).

Results of ${ }^{51} \mathrm{Cr}$ release tests. The results of chromium release tests in which 2F11 and RMA cells were used as HPV16 E7positive and -negative cells, respectively, are shown in Fig. 8. Splenocytes taken at the time of challenge from mice immunized with either B1 or B9 cells displayed cytotoxicity for the E7-positive 2F11 cells but not for the E7-negative RMA cells. The activity of splenocytes from B9-immunized mice was somewhat higher. The reactivities of splenocytes from non-immunized animals with either E7-positive or -negative cells were negligible.

\section{Discussion}

In the present study we investigated the properties of genetically modified cell lines derived from the murine HPV16-transformed MK16 cells, which are characterized by downregulation of MHC class I expression. A subline of these cells, 123IA, which is cTK negative, and the HSV TK gene, the product of which enabled easy selection of transduced cells, were used in the present experiments. Cell lines 
expressing MCP-1 (M2/7 cells), B7.1 (B1 cells) and GMCSF (B9 cells) immunostimulatory molecules were compared. We selected cell clones with the highest production of the respective gene products. In some experiments 123IA cells transduced with the HSV TK gene only (C2 cells) were used as a control. The in vitro characterization of these cells indicated that all of them produced significant levels of HSV TK and that the expression of the HPV16 E6 and E7 genes was not markedly altered by the genetic modification. There was only a slight increase in MHC class I molecules at the surface of the gene-modified cells as compared with the MK16 cells. However, the biological properties of the gene-modified cells were markedly altered. The B7.1 expressing cells had lost oncogenicity completely, being comparable with the GMCSF-expressing B9 cells isolated previously. On the other hand, nearly all mice inoculated with MCP-1 expressing cells developed tumours, though markedly later than animals inoculated with the MK16 cells. This indicated that the production of the MCP-1 chemokine was associated with an attenuation of the oncogenic properties of the transduced cells. The drop in oncogenicity in the $\mathrm{C} 2$ cell line which expresses HSV TK only was much smaller, if any, indicating that HSV TK alone did not play an important role in the decrease of oncogenicity. Both GM-CSF and B7.1-producing cells were capable of inducing protection against challenge with the MK16 cells. It may be of interest that the immunization effect was markedly lower when comparable amounts $\left(\sim 10 \mathrm{TID}_{50}\right.$ doses) of other HPV16 transformed cells, TC-1 cells, were used for challenge. These TC-1 cells, expressing MHC class I molecules, should be a better target for cytotoxic $\mathrm{T}$ cells, presumably developing as a result of cross-priming though dendritic cells, than the MHC class I-negative MK16 cells. This suggests the involvement of MHC non-restricted immune mechanisms in the case of MK16 cells. The indispensable role of NK-cell-mediated immunity in related tumour cells with down-regulated MHC class I expression should be also considered as it has recently been indicated (17). Working with another experimental system in mice, Levitsky et al (18) induced protection against challenge with MHC class I-negative cells by immunization with GM-CSF-transduced MHC-class Inegative cells and this effect was independent of $\mathrm{CD}^{+} \mathrm{T}$ cells. On the other hand, we detected MHC class I molecules in MK16-induced tumours (11). This might be due to endogenous interferon $\gamma$ production. Thus, it is possible that both MHC class I-restricted and non-restricted mechanisms were involved and contributed to the results obtained. The results of the challenge with the two cell lines could also be influenced by differences in their antigenic 'make-up'. It has been shown in other cancer systems (e.g. melanoma or chronic myeloid leukaemia) that an overexpression of proteins of cellular origin may play an important role in anti-tumour immune responses. As reported elsewhere immunization with killed (irradiated) MK16 and TC-1 cells failed to induce cross-protection (19). Those and the present data may reflect the involvement of such antigens. On the other hand, immunization with an HPV16 E7 DNA vaccine is capable of inducing protection against both TC-1 (20) and MK16 (21), demonstrating the significance of the virus-derived antigens in these cells in inducing protection.

One might be inclined to conclude that the complete loss of oncogenicity by the GM-CSF-producing B9 cells and by the B7.1-producing B1 cells, its reduction in the case of the MCP-1-producing M2/7 cells, and the differences in the immunogenicity of B9 and B1 cells are associated with the nature of the immunostimulatory factors produced by the gene-modified cells. The biological effects of the factors involved are complex and varied (reviewed in refs. 22-25) and there is much evidence suggesting that the present GM$\mathrm{CSF}>\mathrm{B} 7.1>\mathrm{MCP}-1$ efficacy gradient is something that might be easily explained and, in fact, expected. Still, the present data should be interpreted cautiously. Only one clone of each subline, with a defined production of the respective immunostimulatory factors was analyzed. It is known from previous studies that the amount of the respective chemokines or cytokines may be of critical importance for the outcome of the immunization/challenge experiments. For more definite conclusions to be drawn a set of clones with varying levels of production should be investigated. Thus, the present results merely offer a lead for further experiments aiming for a better understanding of how tumours evade immune control.

\section{Acknowledgements}

We thank Mrs. V. Navrátilová for her technical assistance. This study was supported by grant no. NR/9075-3 of the Internal Grant Agency of the Ministry of Health of the Czech Republic.

\section{References}

1. Humphreys RE, Hillman GG, von Hofe E and Xu M: Forcing tumor cells to present their own tumor antigens to the immune system: a necessary design for an efficient tumor immunotherapy. Cell Mol Immunol 1: 180-185, 2004.

2. Kagaya T, Nakamoto Y, Sakai Y, Tsuchiyama T, Yagita H, Mukaida N and Kaneko S: Monocyte chemoattractant protein-1 gene delivery enhances antitumor effects of herpes simplex virus thymidine kinase/ganciclovir system in a model of colon cancer: Cancer Gene Ther 13: 357-366, 2006.

3. Mikyskova R, Indrova M, Simova J, Jandlova T, Bieblova J, Jinoch P, Bubenik J and Vonka V: Treatment of minimal residual disease after surgery or chemotherapy in mice carrying HPV16associated tumours: Cytokine and gene therapy with IL-2 and GM-CSF. Int J Oncol 24: 161-167, 2004.

4. Indrova M, Bubenik J, Mikyskova R, et al: Chemoimmunotherapy in mice carrying HPV16-associated, MHC class $\mathrm{I}^{+}$and class $\mathrm{I}^{-}$ tumours: Effects of CBM-4A potentiated with IL-2, IL-12, GM$\mathrm{CSF}$ and genetically modified tumour vaccines. Int $\mathrm{J}$ Oncol 22 : 691-695, 2003.

5. Indrova M, Bubenik J, Mikyskova R, et al: Tumour-inhibitory and antimetastatic effects of IL-2 in mice carrying MHC class $\mathrm{I}^{-}$ tumours of HPV16 origin. Int J Oncol 20: 643-646, 2002.

6. Janouskova O, Nellessen T, Stokrova J, Jinoch P and Smahel M: Delivery of recombinant adeno-associated virus by jet injection. Int J Mol Med 12: 687-691, 2003.

7. Jinoch P, Zak R, Janouskova O, et al: Immunization with live HPV16-transformed mouse cells expressing the herpes simplex thymidine kinase and either GM-CSF or IL-2. Int J Oncol 23: 775-783, 2003.

8. Smahel M, Sobotkova E, Bubenik J, et al: Metastatic MHC class I-negative mouse cells derived by transformation with human papillomavirus type 16. Br J Cancer 84: 374-380, 2001.

9. Lin KY, Guarnieri FG, Staveley-O'Carroll KF, Levitsky HI, August JT, Pardoll DM and Wu TC: Treatment of established tumors with a novel vaccine that enhances major histocompatibility class II presentation of tumor antigen. Cancer Res 56: 21-26, 1996.

10. Janouskova O, Sima $P$ and Kunke D: Combined suicide gene and immunostimulatory gene therapy using AAV-mediated gene transfer to HPV16 transformed mouse cell: Decrease of oncogenicity and induction of protection. Int J Oncol 22: 569-577, 2003. 
11. Mikyskova R, Bubenik J, Vonka V, Smahel M, Indrova M, Bieblova J, Simova J and Jandlova T: Immune escape phenotype of HPV16-associated tumours: MHC class I expression changes during progression and therapy. Int J Oncol 26: 521-527, 2005.

12. Schwarz E, Freese UK, Gissmann L, Mayer W, Roggenbuck B, Stremlau A and zur Hausen H: Structure and transcription of human papillomavirus sequences in cervical carcinoma cells. Nature 314: 111-114, 1985.

13. Kitasato H, Vonka R, Bostik P, Hamsikova E, Sobotkova E, Smahel M and Vonka V: Properties of Syrian hamster cells transformed by human papillomavirus type 16. Acta Virol 40: 281-288, 1996.

14. Ljunggren HG, Paabo S, Cochet M, Kling G, Kourilsky P and Karre K: Molecular analysis of H-2-deficient lymphoma lines. Distinct defects in biosynthesis and association of MHC class I heavy chains and beta 2-microglobulin observed in cells with increased sensitivity to NK cell lysis. J Immunol 142: 2911-2917, 1989.

15. Speidel K, Osen W, Faath S, Hilgert I, Obst R, Braspenning J, Momburg F, Hammerling GJ and Rammensee HG: Priming of cytotoxic $\mathrm{T}$ lymphocytes by five heat-aggregated antigens in vivo: conditions, efficiency, and relation to antibody responses. Eur J Immunol 27: 2391-2399, 1997.

16. Kingston RE, Chen C, Okayama H and Rose JK: Transfection of DNA into eucaryotic cells. Calcium phosphate transfection. In: Current Protocols in Molecular Biology. Ausubel FM, Brent R, Kingston RE, Moore DD, Seidman JG, Smith JA and Struhl K (eds). John Wiley and Sons, Inc., pp9.1.1-9.1.11, 1987.

17. Reinis M, Simova J, Indrova M, Bieblova J, Pribylova H, Moravcova S, Jandlova T and Bubenik J: Immunization with MHC class I-negative but not -positive HPV16-associated tumour cells inhibits growth of MHC class I-negative tumours. Int J Oncol 30: 1011-1017, 2007.
18. Levitsky HI, Lazenby A, Hayashi RJ and Pardoll DM: In vivo priming of two distinct antitumor effector populations: the role of MHC class I expression. J Exp Med 179: 1215-1224, 1994.

19. Simova J, Mikyskova R, Vonka V, Bieblova J, Bubenik J and Jandlova T: MHC class I+ and class I- HPV16-associated tumours expressing the E7 oncoprotein do not cross-react in immunization/ challenge experiments. Folia Biol 49: 230-234, 2003.

20. Ji H, Wang TL, Chen CH, Pai SI, Hung CF, Lin KY, Kurman RJ, Pardoll DM and Wu TC: Targeting human papillomavirus type 16 E7 to the endosomal/lysosomal compartment enhances the antitumor immunity of DNA vaccines against murine human papillomavirus type 16 E7-expressing tumors. Hum Gene Ther 20: $2727-2740,1999$.

21. Rittich S, Duskova M, Mackova J, Pokorna D, Jinoch P and Smahel M: Combined immunization with DNA and transduced tumor cells expressing mouse GM-CSF or IL-2. Oncol Rep 13: 311-317, 2005

22. Flies DB and Chen L: Modulation of immune response by B7 family molecules in tumor microenvironments. Immunol Invest 35: 395-418, 2006.

23. Thompson JA, Dissanayake SK, Ksander BR, Knutson KL, Disis ML and Ostrand-Rosenberg S: Tumor cells transduced with the MHC class II Transactivator and CD80 activate tumor-specific CD4+ T cells whether or not they are silenced for invariant chain. Cancer Res 66: 1147-1154, 2006

24. Choi KJ, Kim JH, Lee YS, et al: Concurrent delivery of GM-CSF and B7-1 using an oncolytic adenovirus elicits potent antitumor effect. Gene Ther 13: 1010-1020, 2006.

25. Eager R and Nemunaitis J: GM-CSF gene-transduced tumor vaccines. Mol Ther 12: 18-27, 2005. 